\title{
Establishing murine models of hepatocellular carcinoma using NOD/SCID, nude and Balb/c mice: An initial comparative study
}

\author{
Nghia Minh Do ${ }^{1,2,3}$, Sinh Truong Nguyen ${ }^{1,2,3}$, Phuc Vo Hong ${ }^{1,2,3}$, Trinh Thi-Phuong Ngo ${ }^{1,3}$, Kiet Dinh Truong ${ }^{4}$, \\ Phuc Van Pham ${ }^{1,2,3,5, *(\mathbb{1}}$
}

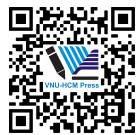

Use your smartphone to scan this QR code and download this article

${ }^{1}$ Stem Cell Institute, University of Science Ho Chi Minh City, Viet Nam

${ }^{2}$ Cancer Research Laboratory, University of Science Ho Chi Minh City, Viet Nam

${ }^{3}$ Viet Nam National University Ho Chi Minh City, Viet Nam

${ }^{4}$ Medical Genetic Institute, Ho Chi Minh City, Viet Nam

${ }^{5}$ Laboratory of Stem Cell Research and Application, University of Science Ho Chi Minh City, Viet Nam

\section{Correspondence}

Phuc Van Pham, Stem Cell Institute University of Science Ho Chi Minh City, Viet Nam

Cancer Research Laboratory, University of Science Ho Chi Minh City, Viet Nam

Viet Nam National University Ho Chi Minh City, Viet Nam

Laboratory of Stem Cell Research and Application, University of Science Ho Chi Minh City, Viet Nam

Email: pvphuc@hcmuns.edu.vn; phucpham@sci.edu.vn

\section{History}

- Received: 15 January 2020

- Accepted: 01 March 2020

- Published: 19 March 2020

DOI : 10.32508/stdj.v23i1.1767

\section{Check for updates}

\section{Copyright}

(๑) VNU-HCM Press. This is an openaccess article distributed under the terms of the Creative Commons Attribution 4.0 International license.

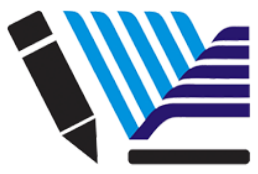

VNU-HCM Press

\begin{abstract}
Introduction: Hepatocellular carcinoma $(\mathrm{HCC})$ is one of the leading causes of cancer mortality in the world. Therefore, more and more studies are developing novel therapies to treat this disease. The pre-clinical trials on animals are a vital step to evaluate the efficacy as well as side effects of these novel therapies. Hence, this study aimed to develop the murine model of HCC using 3 kinds of mice: NOD/SCID, nude and Balb/c mice. Methods: HCC cell line HepG2 was used in this study. They were injected into 3 kinds of mice: NOD/SCID, nude and Balb/c mice at three doses: $5 \times 10^{6}, 2.5 \times 10^{6}$, and $1.25 \times 10^{6}$ cells. Tumor size and body weight of the mice were recorded daily. To confirm these tumors in mice as malignant tumors, they were removed and analyzed by histopathology. Results: The results showed that in nude mice, the tumors appeared after 1 day of injection and could be detected by the naked eye; they continuously developed until the end of the study. In NOD/SCID mice, the tumors could be detected by the naked eye after 3 days of injection; their sizes also increased until the end of the experimental study. Meanwhile, in Balb/c mice, although the tumors could be observed by the naked eye on the $3^{\text {rd }}$ day after cell injection, they regressed and markedly disappeared after 30 days. The dose of $5 \times 10^{6}$ cells per mouse induced the largest tumors $(1.2 \mathrm{~cm}$ in diameter) in NOD/SCID mice. The histopathological analysis showed that the tumors collected from nude and NOD/SCID mice also displayed the poorly differentiated malignant carcinoma with muscle tissue invasion. Conclusion: Both nude and NOD/SCID mice are appropriate for future studies to establish HCC murine models using HepG2 injection.

Key words: Hepatocellular carcinoma, HepG2, cancer, NOD/SCID mice, NUDE mice, BALB/c mice, HCC model
\end{abstract}

\section{INTRODUCTION}

Primary liver cancer begins with abnormally proliferating liver cells. Some common types of primary liver cancer and variants include: hepatocellular carcinoma (HCC), fibrolamellar carcinoma, sclerosing hepatic carcinoma, cholangiocarcinoma, epithelioid hemangioendothelioma, and hepatoblastoma. Remarkedly, HCC accounts for $85-90 \%$ of all primary malignant tumors. Liver cancer is more common in men than in women. In 2012, there were 782,500 new cases in the world and 745,500 deaths, with China accounting for about $50 \%$ of total mortality cases. The highest rates of liver cancer are seen in East and Southeast Asia, North and West Africa, the lowest region of South-Central Asia, and Europe ${ }^{1}$.

One of the most frequently used methods of cancer research is xenograft transplantation which allows for observations of growth, invasive process, and metastases of human tumors. In xenograft models, the inhibition of alloimmunization is extremely important and, therefore, immunocompromised mice are essen- tial for use. In 1966, the first human cell line to be successfully transplanted in nude mice was able to be developed and maintained ${ }^{2}$. Nude mice carry the mutation in the FoxN1 gene that causes T-cell deficiency but still have intact B-cell and innate immune systems $^{3}$. In 1980, Makino and colleagues created a line of non-obese diabetic mice (NOD) mice with weakened innate immune systems. In 1983, a mutation in the Prkdc ${ }^{S C I D}$ gene coding for protein kinase catalyzing the activation of DNA led to a severely weakened lymphatic system in CB-17 mice ${ }^{4}$. Crossbreeding NOD and SCID mice led to the creation of mice with deficiencies in both the innate and adaptive immune systems; this model, called the NOD/SCID mouse model, has been a useful model for human cell transplantation over the past two decades.

Nowadays, more than $80 \%$ of drug development studies show the potential of new drug candidates for cancer treatment, with positive results in animal studies. However, there is insufficient or no evidence to show their effectiveness in humans ${ }^{5}$. This is mainly 
due to the use of preclinical models that have not yet simulated human physiological and pathological processes. These models have many characteristics that differ from humans in terms of the physiology of organs, cellular dynamics, and regulatory proteins. Therefore, one of the major challenges of medical development is to create more humanized models that can easily allow the transplantation of human cell systems into animals. Humanized mouse models can be created by incorporating human genes into mice by transplanting functional cells, tissues or organs ${ }^{6}$.

In this study, three mouse strains, including nude, NOD/SCID and Balb/c mice, were subcutaneously injected with hepatocellular carcinoma. The aims were to investigate the ability of each mouse model to induce tumor-carry and tumor formation; as well, tumor structure was evaluated by histochemical staining. To our understanding, this study herein is the first to compare the use of these three mouse groups in liver cancer research in vivo.

\section{MATERIALS \& METHODS}

\section{Animals}

Female $\mathrm{Balb} / \mathrm{c}$, nude and NOD/SCID mice, 6-8 weeks old and 20-24 g in weight, were purchased from Jax Laboratory and kept at the Stem Cell Institute (University of Science, Ho Chi Minh City, Viet Nam). The mice were maintained in a clean environment with humidity of about $60 \%$ and temperature of $25^{\circ} \mathrm{C}$. The light system was automatically controlled at every 12 hour interval.

\section{Hepatocellular carcinoma HepG2 cell line}

Hepatocellular carcinoma HepG2 cells (Manassas, VA, USA) were thawed and expanded. They were cultured in Dulbecco's Modified Medium/Nutrient Mixture F12 (DMEM/F12; Thermo Fisher Scientific, Waltham, MA), with $10 \%$ fetal bovine serum (FBS; Sigma-Aldrich, St. Louis, MO).

\section{Injection of HepG2 cells into mice}

Mice were divided into 4 groups, with each group consisting of 3 individuals. Mice were subcutaneously injected with $5 \times 10^{6}, 2.5 \times 10^{6}$, or $1.25 \times 10^{6}$ cells that were suspended in $100 \mu \mathrm{L}$ of DMEM/F12 per site; control mice were injected with $100 \mu \mathrm{L}$ of DMEM/F12 (Figure 1). All manipulations on mice were approved by the Institutional Ethics Committee of the Stem Cell Institute.

\section{Evaluation of mouse tumor size}

After injection of HepG2 cells, all mice were checked for tumor size in a fixed daily time frame (once per day). A straight ruler, with a minimum division of 1 $\mathrm{mm}$, was used to measure the size of the tumor. This experiment was performed for 30 days, or until the mice died, or until tumor size exceeded $2 \mathrm{~cm}$.

\section{Histopathological analysis}

Tumors were collected from mice and fixed in $4 \%$ formaldehyde overnight. Then, they were soaked in $30 \%$ sucrose solution until they completely sank. Tumor blocks were then created in OCT (Optimal Cutting Tissue) (Thermo Fisher Scientific, Waltham, MA, USA). The tissue sections were sliced, stained and analyzed at Cho Ray Hospital (Ho Chi Minh City, Viet Nam).

\section{RESULTS}

\section{Tumor formation}

The tumors formed after 5 days (with a probability of $100 \%)$ in the three groups injected with HepG2 cells. The control injection site did not show any appearance of tumor nor any inflammation, ulcer or necrosis. In the NOD/SCID and nude mouse groups, at the $10^{\text {th }}$ day after tumor cell injection, there was development of large and bulging tumors under the skin; around the tumor area, a vein appeared to be directed towards the tumor. The tumor size was seen to increase continuously in both the nude and NOD/SCID mice until the $30^{\text {th }}$ day. On the other hand, after the $10^{\text {th }}$ day, the tumor size of the Balb/c group decreased (Figure 2).

After 30 days, in the NOD/SCID mice, the largest tumor size was recorded at a diameter of $1.2 \mathrm{~cm}$ with the dose concentration of $5 \times 10^{6}$ cells; at the concentrations of $2.5 \times 10^{6}$ and $1.25 \times 10^{6}$ cells, tumors arose of similar size (about a $1 \mathrm{~cm}$ diameter). Tumors in nude mice were recorded from the first day after injection; their sizes were $0.6,0.4$, and $0.2 \mathrm{~cm}$ at the concentrations of $5 \times 10^{6}, 2.5 \times 10^{6}$, and $1.25 \times 10^{6}$ cells, respectively. The tumors maintained growth and continuously grew to $1.3,1$, and $0.7 \mathrm{~cm}$, respectively. By contrast, after 5 days, tumors in the Balb/c group began to adapt and grow. The largest tumor size was recorded using a dose concentration of $5 \times 10^{6}$ cells; the tumor diameter was about $0.7 \mathrm{~cm}$. For the other two concentrations $\left(2.5 \times 10^{6}\right.$ and $1.25 \times 10^{6}$ cells $)$, the mean tumor size was $0.5 \mathrm{~cm}$ and $0.4 \mathrm{~cm}$, respectively. After day 10, tumor size began to decline and some tumors appeared to undergo necrosis and the tumors dissolved. In addition, external observations 


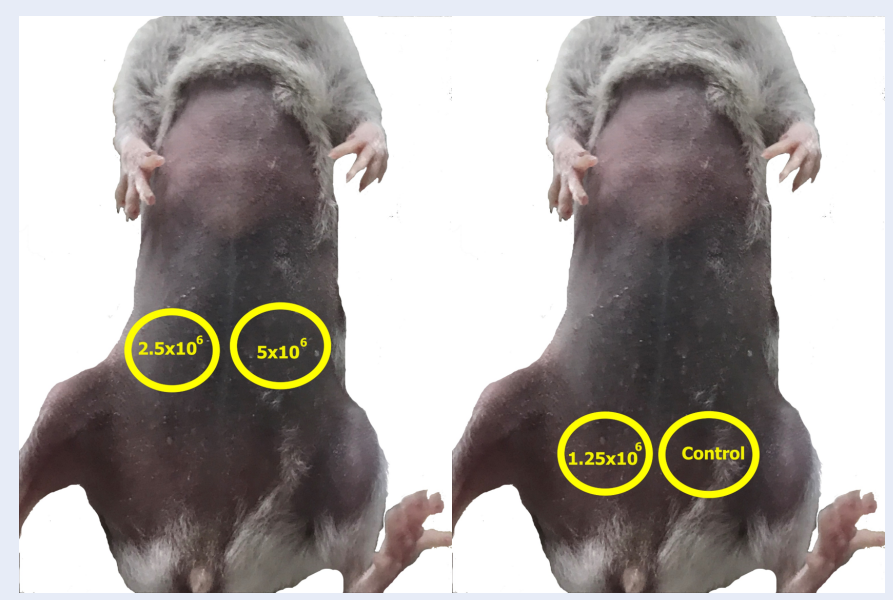

Figure 1: Cell injection sites in NOD/SCID and NUDE mice.

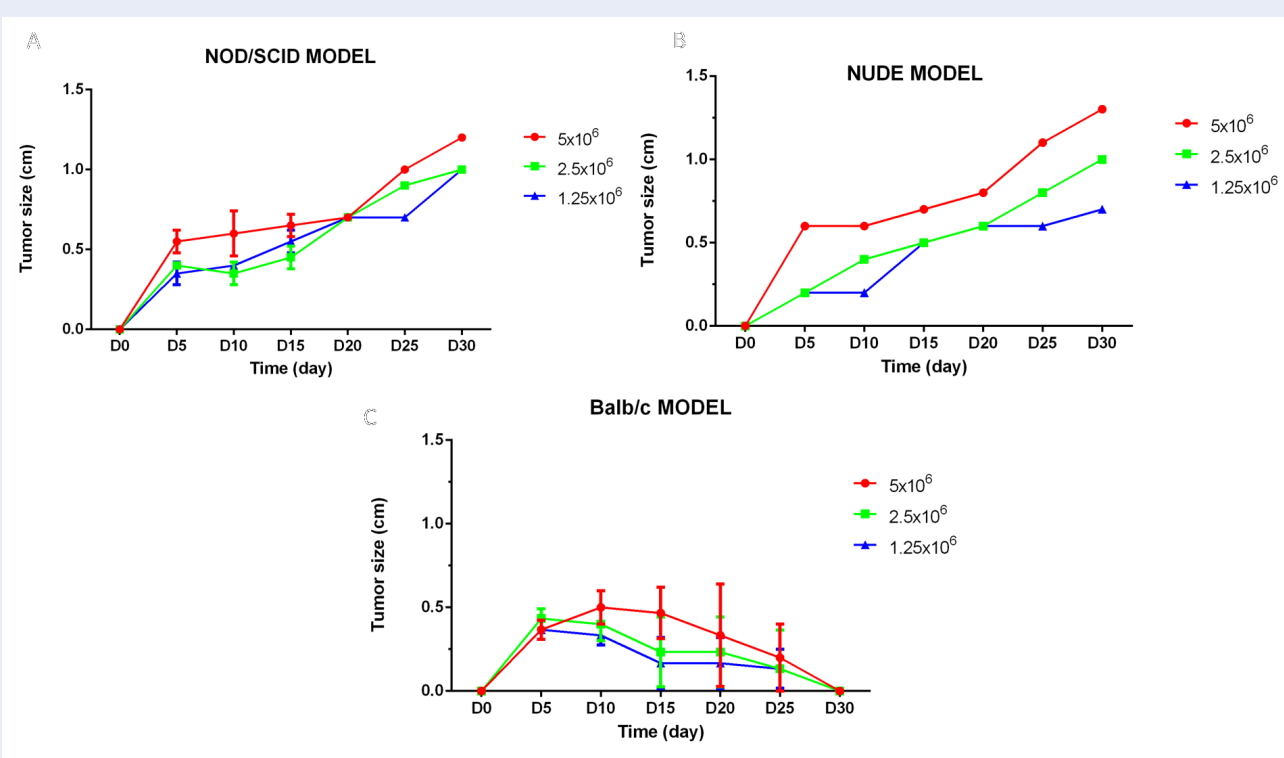

Figure 2: Size of tumor of HCC mice model. (A) Size of tumor of HCC NOD/SCID mice model; (B) Size of tumor of HCC NUDE mice model; (C) Size of tumor of HCC Balb/c micemodel; Mice were injected HepG2 cells with three concentrations include $5 \times 10^{6}, 2.5 \times 10^{6}, 1.25 \times 10^{6}$.

also showed that the mice did not show signs of ruffled feathers, anorexia or abnormal aggression; indeed, the percentage of mice living until the end of the experiment was $100 \%$.

In NOD mice, the mean tumor weight reached 0.4315 $\pm 0.0999,0.3067 \pm 0.0677$, and $0.23 \pm 0.0003 \mathrm{~g}$, when injected with hepatocellular carcinoma at a concentration of $5 \times 10^{6}, 2.5 \times 10^{6}$, and $1.25 \times 10^{6}$, respectively (Figure 3 ). In addition, for the injection dose of $2.5 \times 10^{6}$ cells, the injection resulted in 2 small metastatic cancer sites found $1 \mathrm{~cm}$ away from the orig- inal tumor injection site. Similarly, in the nude mice, the tumor weights reached $0.1959,0.2773$, and 0.0948 $\mathrm{g}$ at the sites injected with hepatocellular carcinoma cells at a concentrations of $5 \times 10^{6}, 2.5 \times 10^{6}$, and 1.25 $\mathrm{x} 10^{6}$, respectively. In addition, two metastatic sites of cancer were recorded. At the control injection site (DMEM injection), however, no abnormalities were reported.

The cell mass was observed to be a large, round solid tumor. In addition, the heterogeneous tumor was divided into many parts, with a rough surface separated 


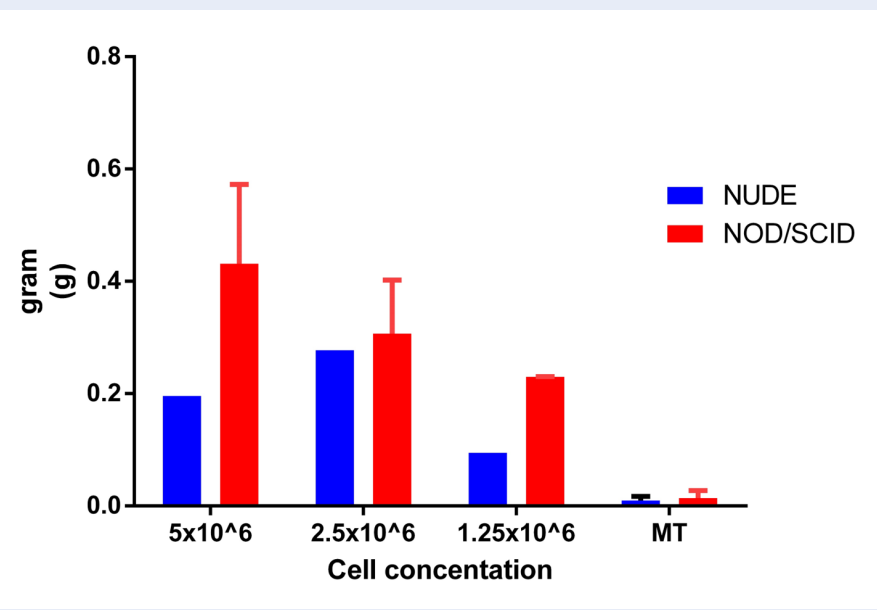

Figure 3: Tumorfomation weight in HCC model mice. Abbreviations: MT: Metatasis Tumors, HCC: Hepatocellular carcinomar

from the surrounding tissues. Small tumors (believed to have been caused by metastases) appeared about 1 $\mathrm{cm}$ away from the main tumor site. The anatomical results also indicated the presence of many blood vessels around the tumor (Figure 4).

\section{H\&E staining}

Tumor anatomical surgery showed the tissue structure of the cancer contained polymorphic cells arranged in clusters with invasive structure. The cancer cells had an abnormal dividing nucleus. The cells in the tumor center were necrotic or poorly developed, such that nucleus showed little to no staining with hematoxylin dye. This is a characteristic of poorly differentiated epithelial tumors with invasive muscle tissue (Figure 5).

\section{DISCUSSION}

This report aims to, firstly, demonstrate a suitable mouse model for hepatocellular carcinoma research. All injected mice, including immune-deficient and normal mice, formed tumors at the injection site. From this, it can be seen that HepG2 cells have the ability to adapt and develop well in the in vivo environment of mice. However, there were differences between the mouse strains tested. For experiments in immunodeficient mice, the tumors adapted and developed well, and no elimination nor inflammation took place. The injected HepG2 cells showed the ability to construct tumors between abdominal skin and muscle layer in the HepG2 cell transplantation positions and could adapt to the mouse body through the process of forming new blood vessels. The angiogenesis involves the proliferation of capillaries and the rebuilding of existing blood vessels. In particular, the NOD/SCID mice group results provided evidence of continual development/increase in tumor size and weight. On the other hand, the Balb/c group of mice showed a decline in tumor size and, thus, the mouse strain was unsuitable for the HCC model.

In experiments by Yu-Huei Liu et al., the authors induced liver cancer tumors in NOD/SCID mice by subcutaneous injection method, using $5 \times 10^{6}$ cells/mouse as the dose of cell injection ${ }^{7}$. Honghai Xia used a lower cell dose of $10^{6}$ cells/mouse because the experiments took longer (90 days $)^{8}$. Almost the experiments conducted show the adaptiveness of HepG2 cells in NOD/SCID mice, the tumors formed at the xenograft position, developed, and were maintained as stable. In the experiments using a dose concentration of $5 \times 10^{6}$ cells, after 15 days of injection, the tumors had an average diameter of 0.6 $\mathrm{cm}^{7,9}$. In this experiment, we created a model with subcutaneous injection at a cell dose of $5 \times 10^{6}$; the resulting tumor size at day 15 was $0.65 \pm 0.05 \mathrm{~cm}$. It was demonstrated that the procedures used in the study, combined with the specific care conditions in Vietnam, could allow HepG2 cells to adapt well in NOD/SCID mice.

Histopathological results of the tumors showed that the development of hepatocellular carcinoma cells in the mouse models were accompanied by characteristics such as clumping of polymorphic cells, formation of an invasive strip, and establishment of a necrotic area inside the tumors. In addition, the cells showed an unequally divided nucleus and abnormally small size. In particular, it is clearly observed that cells of 


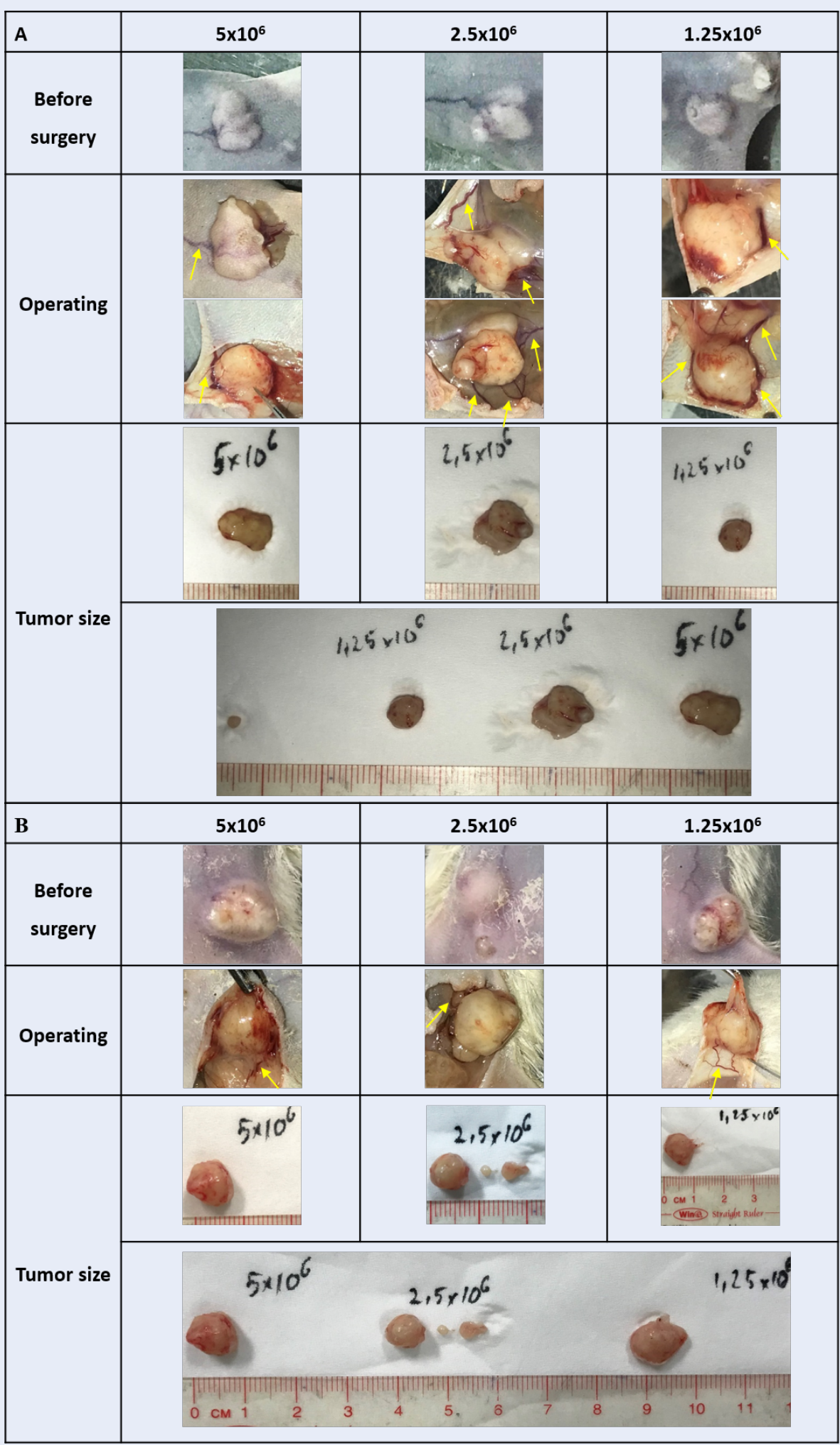

Figure 4: Image of surgery tumor on mice model. (A) NUDE mice model; (B): NOD/SCID mice model. The arrows indicate the blood vessels around the tumor. 

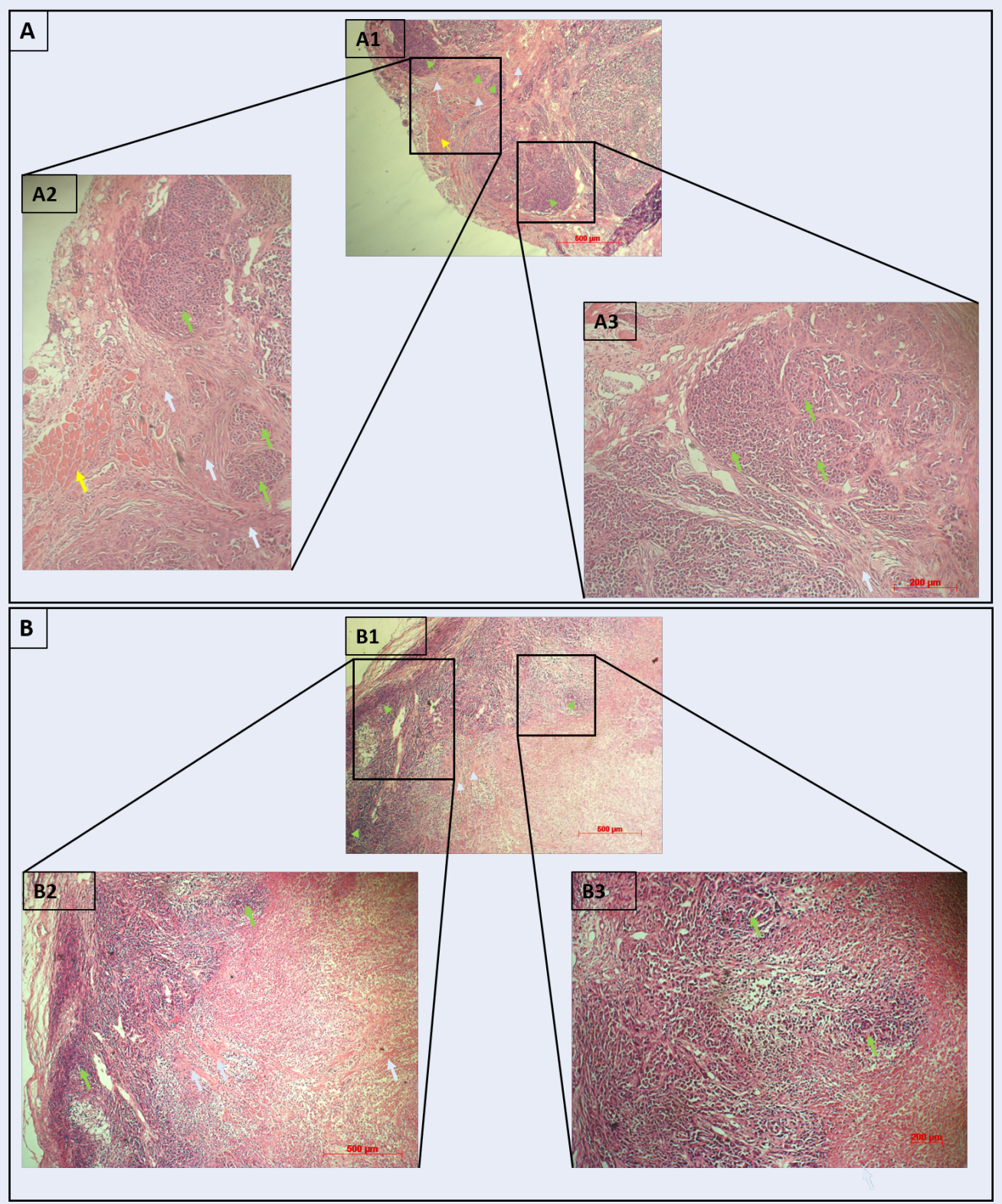

Figure 5: Result of H\&E staining in HepG2 tumor sample of immunodeficient mice. Tumor sample of NUDE mice (A, A1, A2), and NOD/SCID mice (B, B1, B2); green arrows indicate evidence of cancer cell clumps, yellow arrows identify the areas of muscle tissue, gray arrows expose the fibrosis tissues.

the tumors had abnormal nuclei, ambiguous boundaries, invasive surrounding tissues, and proliferating blood vessels. Taken together, these observations show that HepG2 liver cancer cells, indeed, grew well in NOD/SCID mice. The resulting tumors, when imaged, had morphological and histological structures similar to those observed by Lekshmi R. Nath et al. in their NOD/SCID mouse model ${ }^{10}$. The results are also consistent with the research of Qiang-Bo Zhang et al., who created a Balb/c mouse model carrying the
HepG2 cell tumor to study the activity of Sorafenib ${ }^{11}$. From these, it is evident to conclude that tumors in the NOD/SCID mouse model are established with the typical histological structures of xenografted HepG2 cell tumors in immunodeficient mice. The acquired tumors are poorly differentiated epithelial tumors that have invasive muscle tissues.

However, the research herein does have limitations. Our study was conducted with finite funding and, thus, the experiment with the group of immunode- 
ficient mice was not repeated. In order to more accurately assess the potential of immunodeficient mice in an HCC mouse model, the experiments need to be repeated at least twice and evaluated using more intensive methods.

\section{CONCLUSION}

HepG2 cells were xenografted into immunodeficient mice (nude and NOD/SCID); notably in NOD/SCID mice, the tumors formed at a rate of $100 \%$, were maintained, and grew in size during the experimental study. Moreover, tumor histology results from these groups of mice also showed that these tumors were malignant tumors. On the other hand, in $\mathrm{Balb} / \mathrm{c}$ mice, the tumors were present from the $5^{\text {th }}$ to $10^{\text {th }}$ day after injection, then gradually disappeared. Therefore, it can be concluded that immunodeficient mice, especially the NOD/SCID model, is advisable and suitable for the generation a mouse model of human liver cancer by xenograft transplantation.

\section{ABBREVIATIONS}

HCC: Hepatocellular Carcinoma

H\&E: Hematoxylin and Eosin

OCT: Optimal Cutting Tissue

\section{ACKNOWLEDGMENTS}

This work was supported by the Vietnam National University, Ho Chi Minh City, Vietnam, under grant A2015-18-01.

\section{CONFLICT OF INTEREST}

The authors report no conflicts of interest in this work.

\section{AUTHORS' CONTRIBUTION}

All authors equally contributed in this work and approved the final version of manuscript for submission.

\section{REFERENCES}

1. McGlynn K, Meyts ERD, Stang A. Cancer Epidemiology and Prevention: Testicular Cancer. In: Cancer Epidemiology and
Prevention. Oxford University Press. 2017;p. 1019-1028. Available from: https://doi.org/10.1093/oso/9780190238667. 003.0054 .

2. Flanagan S. 'Nude', a new hairless gene with pleiotropic effects in the mouse. Genetics Research. 1966;8(3):295309. PMID: 5980117. Available from: https://doi.org/10.1017/ S0016672300010168.

3. Ganick DJ, Sarnwick RD, Shahidi NT, Manning DD. Inability of intravenously injected monocellular suspensions of human bone marrow to establish in the nude mouse. International Archives of Allergy and Immunology. 1980;62(3):330333. PMID: 6993372. Available from: https://doi.org/10.1159/ 000232530 .

4. Bosma GC, Custer RP, Bosma MJ. A severe combined immunodeficiency mutation in the mouse. Nature. 1983;301(5900):527. PMID: 6823332. Available from: https: //doi.org/10.1038/301527a0.

5. Perrin S. Preclinical research: Make mouse studies work. Nature . 2014;507(7493):423-425. PMID: 24678540. Available from: https://doi.org/10.1038/507423a.

6. Brehm MA, Shultz LD, Greiner DL. Humanized mouse models to study human diseases. Current opinion in endocrinology, diabetes, and obesity. 2010;17(2):120. PMID: 20150806. Available from: https://doi.org/10.1097/MED.0b013e328337282f.

7. Liu YH, Weng YP, Lin HY, Tang SW, Chen CJ, Liang CJ, et al. Aqueous extract of Polygonum bistorta modulates proteostasis by ROS-induced ER stress in human hepatoma cells. Scientific reports. 2017;7:41437. PMID: 28134285. Available from: https://doi.org/10.1038/srep41437.

8. Xia H, Cao J, Li Q, Lv Y, Jia W, Ren W, et al. Hepatocellular Carcinoma-propagating Cells are Detectable by Side Population Analysis and Possess an Expression Profile Reflective of a Primitive Origin. . Scientific reports. 2016;6:34856. PMID: 27725724. Available from: https://doi.org/10.1038/srep34856.

9. Wu YH, Cao JG, Xiang $\mathrm{HL}$, Xia H, Qin $\mathrm{Y}$, Huang $\mathrm{AJ}$, et al. Recombinant vascular basement-membrane-derived multifunctional peptide inhibits angiogenesis and growth of hepatocellular carcinoma. World journal of gastroenterology: WJG . 2009;15(14):1744. PMID: 19360918. Available from: https: //doi.org/10.3748/wjg.15.1744.

10. Nath LR, Gorantla JN, Thulasidasan AKT, Vijayakurup V, Shah $S$, Anwer $S$, et al. Evaluation of uttroside B, a saponin from Solanum nigrum Linn, as a promising chemotherapeutic agent against hepatocellular carcinoma. Scientific Reports. 2016;6:36318. PMID: 27808117. Available from: https://doi. org/10.1038/srep36318.

11. Q-B Z, H-CS, K-ZZ, Q-A J, Y B, M W, et al. Suppression of natural killer cells by sorafenib contributes to prometastatic effects in hepatocellular carcinoma. PLoS One. 2013;8(2):e55945. PMID: 23409093. Available from: https://doi.org/10.1371/journal. pone.0055945. 